MATHEMATICS OF COMPUTATION

Volume 80, Number 276, October 2011, Pages 2445-2454

S $0025-5718(2011) 02479-8$

Article electronically published on March 18, 2011

\title{
ADDENDA AND CORRIGENDA TO "THE MINKOWSKI QUESTION MARK FUNCTION: EXPLICIT SERIES FOR THE DYADIC PERIOD FUNCTION AND MOMENTS"
}

\author{
GIEDRIUS ALKAUSKAS
}

\begin{abstract}
In this supplement we fully complete the proofs of the statements which were left out or only briefly sketched in the main paper.
\end{abstract}

\section{AdDEndA}

References to equations in [1] are numbered, and equations in this note are indexed by Latin letters. Page and line numbers refer to [1] only.

1.1. Our main Theorem 1.2 depends on the validity of the Theorem 3.1 which we reproduce here.

Theorem 1. There exists the unique function $\mathfrak{X}_{\mathrm{p}}(x)=\mathfrak{X}(\mathrm{p}, x):\{|\mathrm{p}-2| \leq 1\} \times$ $[0, \infty] \rightarrow \mathbb{C} \cup\{\infty\}$, having these properties:

(i) $\mathfrak{X}_{\mathrm{p}}(x)$ satisfies the functional equations

(a)

$$
\begin{aligned}
\mathfrak{X}_{\mathrm{p}}(x+1) & =\frac{\mathfrak{X}_{\mathrm{p}}(x)+1}{\mathrm{p}}, \\
\mathfrak{X}_{\mathrm{p}}\left(\frac{x}{x+1}\right) & =\frac{\mathrm{p} \mathfrak{X}_{\mathrm{p}}(x)}{\mathfrak{X}_{\mathrm{p}}(x)+1}, \\
\mathfrak{X}_{\mathrm{p}}\left(\frac{1}{x}\right) & =\frac{1}{\mathfrak{X}_{\mathrm{p}}(x)} .
\end{aligned}
$$

(ii) For fixed $\mathrm{p} \neq 1, \mathfrak{X}(\mathrm{p}, x):[0, \infty] \rightarrow \mathbb{C}$ is a continuous function, and the image (denote it by $\mathscr{I}_{\mathrm{p}}$ ) is thus a bounded curve; it is contained in the domain $\mathbb{C} \backslash\left\{|z+1| \leq \frac{3}{4}\right\}$.

(iii) For every $\mathrm{p},|\mathrm{p}-2| \leq 1, \mathrm{p} \neq 1$, in some neighborhood of $\mathrm{p}$ there exists the derivative $\frac{\partial}{\partial \mathrm{p}} \mathfrak{X}(\mathrm{p}, x)$, which is a continuous and bounded function for $x \in[0, \infty]$.

(iv) There exist all derivatives $\mathcal{S}_{N}(x)=\left.\frac{\partial^{N}}{\partial \mathrm{p}^{N}} \mathfrak{X}(\mathrm{p}, x)\right|_{\mathrm{p}=1}:[0, \infty) \rightarrow \mathbb{R}$ (the derivatives are taken inside $|\mathrm{p}-2| \leq 1)$. These functions are bounded in any finite interval and continuous for irrational $x$. Moreover, $\mathcal{S}_{N}(x) \ll_{N} x^{N+1}$ for $x \geq 1$, and $\mathcal{S}_{N}(x) \ll_{N} 1$ for $x \in(0,1)$.

Received by the editor July 10, 2010 and, in revised form, August 30, 2010.

2010 Mathematics Subject Classification. Primary 11A55, 26A30, 32A05; Secondary 40A15, 37F50, 11F37.

Key words and phrases. The Minkowski question mark function, the dyadic period function, three-term functional equation, analytic theory of continued fractions, Julia sets, the Farey tree. 
Note a slight difference in a phrasing of a statement in part (iv), as compared with the one given in [1. The proof presented in [1, Section 3, establishes only the parts (i) and (ii), and also the analyticity of $\mathfrak{X}(\mathrm{p}, x)$ in variable $\mathrm{p}$. Here we complete the missing proofs (p. 402, l. 7-15 from above).

1.1.1. (ii). Let $\chi=2+e^{2 \pi i / 3}$. Part (ii) was proven completely in [1], except that an exhibition that the image of the curve $\mathscr{I}_{\mathrm{p}}$ in case $|\mathrm{p}-\chi| \leq 0.19,|\mathrm{p}-2| \leq 1$, is contained outside the disc $|z+1| \leq \frac{3}{4}$, was omitted. This complication arises from the fact that the first column of the table on p. 413 contains infinitely many numbers slightly greater than 0.5 . It was pointed out (p. 402) that value region problems of continued fractions for more general parabolic domains are sufficient to fill this gap. As we will see, this is indeed the case. We need the simplified version of a result by $\mathrm{H}$. S. Wall (the convergence of a corresponding continued fraction was proved in [1]; we need only the value region).

Theorem 2 ([3, Chapter VIII]). Suppose, given complex numbers $z_{\nu} \neq 0, \nu \geq 2$, such that for a certain $\phi,-\pi<\phi<+\pi$,

$$
\left|z_{\nu}\right|-\Re\left(e^{i \phi} z_{\nu}\right) \leq \frac{1}{2} \cos ^{2}\left(\frac{\phi}{2}\right), \quad \nu \geq 2 .
$$

Assume that the following continued fraction converges:

$$
\mathcal{F}=\frac{1}{1+\frac{z_{2}}{1+\frac{z_{3}}{1+\frac{z_{4}}{\ddots}}}}
$$

Then

$$
\left|\mathcal{F}-e^{i \phi / 2} \sec \left(\frac{\phi}{2}\right)\right| \leq \sec \left(\frac{\phi}{2}\right)
$$

Considering the inequality (19) on p. 401, to exhibit that the curve $\mathscr{I}_{\mathrm{p}}$ is contained outside $|z+1| \leq \frac{3}{4}$, we need to show that if $a_{i} \in \mathbb{N}$, then

$$
\left|\mathrm{p}^{-1} W_{a_{1}}^{-1} \mathcal{F}\left(\mathrm{p}, a_{1}, a_{2}, \ldots\right)-1\right| \leq \frac{4}{3}
$$

here $\mathcal{F}\left(\mathrm{p}, a_{1}, a_{2}, \ldots\right)$ is given by the formula $(15), W_{a}(\mathrm{p})=\frac{\mathrm{p}^{a}-1}{\mathrm{p}^{a+1}-\mathrm{p}^{a}}$ and $T_{a, b}(\mathrm{p})=$ $\frac{(p-1)^{2} p^{b}}{\left(p^{a}-1\right)\left(p^{b}-1\right)}$. We will prove the above inequality exactly the same way as in Appendix A.2. All we need are the following lemmas which are obtained by computer calculations. Let $\phi=-0.04$. Then computations show that for all pairs $a, b \in \mathbb{N}$,

$$
\begin{aligned}
\bar{\mu}(a, b) & =\sup _{|\mathrm{p}-2| \leq 1,|\mathrm{p}-\chi| \leq 0.19}\left(\left|T_{a, b}(\mathrm{p})\right|-\Re\left(e^{i \phi} T_{a, b}(\mathrm{p})\right)\right)<0.48<0.4998_{+} \\
& =\frac{1}{2} \cos ^{2}\left(\frac{\phi}{2}\right) .
\end{aligned}
$$

Note that $\sec (\phi / 2)=1.0002_{+}$and $e^{i \phi / 2} \sec (\phi / 2)=1-0.02_{+} i$. Now the inequality (IC) follows from (b) , Lemma A.2 and the following result, which is also the outcome of computer calculations: 
Lemma 1. One has

$$
\sup _{|\mathrm{p}-2| \leq 1,|\mathrm{p}-\chi| \leq 0.19, a \in \mathbb{N},\left|z-1+0.02_{+} i\right| \leq 1.0002_{+}}\left|\mathrm{p}^{-1} W_{a}^{-1}(\mathrm{p}) z-1\right|<1.29 .
$$

(Compare this with Lemma A.3). Similar analysis applies to $\bar{\chi}=2+e^{-2 \pi i / 3}$.

1.1.2. (iv). As is clear from p. 393 and further, any two of the functional equations (a) imply the third. We will use the first and the third one. By the construction, $\mathfrak{X}(1, x)=x$. Let us differentiate these two equations with respect to $\mathrm{p}$ and substitute $\mathrm{p}=1$. Let $\left.\frac{\partial}{\partial \mathrm{p}} \mathfrak{X}(\mathrm{p}, x)\right|_{\mathrm{p}=1}=\mathcal{S}_{1}(x)$, as already defined. Thus, the function $\mathcal{S}_{1}(x)$ satisfies the following functional equations:

$$
\mathcal{S}_{1}(x)=-x^{2} \mathcal{S}_{1}\left(\frac{1}{x}\right) ; \quad \mathcal{S}_{1}(x)=-x+\mathcal{S}_{1}(x-1), \quad x \geq 1 .
$$

Note that this function cannot be continuous for all real $x \geq 0$. Indeed, these functional equations determine the value of $\mathcal{S}_{1}(x)$ for rational $x$ uniquely. Indeed, $\mathcal{S}_{1}(1)=0, \mathcal{S}_{1}(0)=1$. This implies $\mathcal{S}_{1}(n)=-\frac{(n+2)(n-1)}{2}$, and $\mathcal{S}_{1}\left(\frac{1}{n}\right)=\frac{(n+2)(n-1)}{2 n^{2}}$ for $n \in \mathbb{N}$. Hence, $\mathcal{S}_{1}(x)$ is discontinuous at $x=0$ and therefore it is discontinuous at every rational point. Nevertheless, as will be apparent soon, it is continuous for irrational $x$. Let $x=\left[a_{0}, a_{1}, a_{2}, \ldots\right]$ be an irrational number. Let us define $\epsilon_{i}=\left[0, a_{i}, a_{i+1}, \ldots\right], x_{i}=\left[a_{i}, a_{i+1}, \ldots\right]=\frac{1}{\epsilon_{i}}$. Finally, if $x \in(n, n+1), n \geq 1$, let $D(x)=n x-\frac{n(n-1)}{2}$. Then the functional equations (d) imply:

(e) $\mathcal{S}_{1}(x)=-D\left(x_{0}\right)+\epsilon_{1}^{2} D\left(x_{1}\right)-\epsilon_{1}^{2} \epsilon_{2}^{2} D\left(x_{2}\right)+\epsilon_{1}^{2} \epsilon_{2}^{2} \epsilon_{3}^{2} D\left(x_{3}\right)-\cdots$.

This is valid for $x \geq 1$, and also for $x \in(0,1)$, if we define $D(x)=0$ for $x \in(0,1)$. First, we have an estimate:

$$
\epsilon_{i} \cdot \epsilon_{i+1}=\frac{1}{a_{i}+\frac{1}{a_{i+1}+\epsilon_{i+2}}} \cdot \frac{1}{a_{i+1}+\epsilon_{i+2}}=\frac{1}{a_{i}\left(a_{i+1}+\epsilon_{i+2}\right)+1}<\frac{1}{a_{i} a_{i+1}+1} \leq \frac{1}{2} .
$$

Second, if $x=n+\epsilon>1$, then

$$
\frac{D(x)}{x^{2}}=\frac{n(n+\epsilon)-\frac{n(n-1)}{2}}{(n+\epsilon)^{2}} \leq \frac{n+1}{2 n} \leq 1 .
$$

This shows that the series (远) absolutely converges and it is obviously a continuous function for irrational $x$. As an aside, the situation here is completely analogous to the one we encounter considering the classical function $f\left(\frac{p}{q}\right)=\frac{1}{q}$ if $(p, q)=1$, $q \geq 1$, and $f(x)=0$ for $x \in \mathbb{R} \backslash \mathbb{Q}$. The latter is continuous precisely for irrational $x$. In our case, if a rational or irrational point $y$ approaches irrational $x$, then their first $M$ partial quotients necessarily coincide and $M \rightarrow \infty$ as $y \rightarrow x$. Thus, the solution to (d) is the unique function, therefore it arises precisely from $\mathfrak{X}_{\mathrm{p}}(x)$. Since $D(x) \sim \frac{1}{2} x^{2}$, this proves the part (iv) in case $N=1$. The part (iv) is needed to show that $G(\mathrm{p}, z)$ has all partial $\mathrm{p}$-derivatives at $\mathrm{p}=1$. The formula on $\mathrm{p}$. 404, l. 14 from below, gives

$$
\left.\frac{\partial}{\partial \mathrm{p}} G(\mathrm{p}, z)\right|_{\mathrm{p}=1}=-\int_{0}^{\infty} \frac{\mathcal{S}_{1}(x)}{(x+1-z)^{2}} \mathrm{~d} F(x) .
$$

Since $2^{-x} \asymp 1-F(x)$, as $x \rightarrow \infty, \mathrm{d} F(x)$ "kills" every positive power of $x$. Moreover, $\mathcal{S}_{1}(x)$ is continuous for irrational $x$. According to the Lebesgue criterion 
for Riemann-Stieltjes integrability, a function on a compact interval is RiemannStieltjes $\mu$-integrable if it is bounded and it is continuous almost $\mu$-everywhere. Since rational numbers form a countable set, $\mathcal{S}_{1}$ is continuous almost $F$-everywhere, the above integral exists in a Riemann-Stieltjes sense and the expression on the lefthand side is properly defined for $z \notin(1, \infty)$.

For an arbitrary $N$ we prove (iv) by induction. Let us differentiate the first and the third equations of (国) $N$ times and substitute $\mathrm{p}=1$. We get

$$
\begin{aligned}
N \mathcal{S}_{N-1}(x+1)+\mathcal{S}_{N}(x+1) & =\mathcal{S}_{N}(x), \\
\mathcal{S}_{N}(x) \frac{1}{x}+\sum_{\ell=1}^{N-1}\left(\begin{array}{c}
N \\
\ell
\end{array}\right) \mathcal{S}_{\ell}(x) \mathcal{S}_{N-\ell}\left(\frac{1}{x}\right)+\mathcal{S}_{N}\left(\frac{1}{x}\right) x & =0 .
\end{aligned}
$$

By induction, the middle sum is $\ll x^{N}$ if $x \geq 1$. All the remaining steps are the same as in case $N=1$. We note that the validity of part (iv) assures that the series in Corollary 1.4 converges faster than $\sum_{n} n^{-M}$ for any $M>1$.

1.1.3. (iii). As before, we use the first and the third functional equations (国). Let $\frac{\partial}{\partial \mathrm{p}} \mathfrak{X}(\mathrm{p}, x)=u(x)$, which does exist, as was shown in [1]. Thus, this function satisfies

$$
\begin{aligned}
\mathrm{p} u(x) & =-\mathfrak{X}_{\mathrm{p}}(x)+u(x-1), \quad x \geq 1, \\
u(x) & =-\mathfrak{X}_{\mathrm{p}}^{2}(x) u\left(\frac{1}{x}\right) .
\end{aligned}
$$

As can be seen, unlike in the case $p=1$, the solution to these functional equations for $|\mathrm{p}-2| \leq 1, \mathrm{p} \neq 1$, is a continuous function. Truly, using properties of the function $\mathfrak{X}_{\mathrm{p}}(x)$ from $\mathrm{p}$. 393, we get $u(1)=0, u(0)=1$, and using the formula from p. $402,1.12$ from below, we get

$$
\begin{aligned}
u(n) & =-\frac{1}{\mathrm{p}} \mathfrak{X}_{\mathrm{p}}(n)-\frac{1}{\mathrm{p}^{2}} \mathfrak{X}_{\mathrm{p}}(n-1)-\cdots-\frac{1}{\mathrm{p}^{n-1}} \mathfrak{X}_{\mathrm{p}}(2)+\frac{1}{\mathrm{p}^{n-1}} u(1) \\
& =-\sum_{i=2}^{n} \frac{\mathrm{p}+\mathrm{p}^{i-1}-2}{\mathrm{p}^{n}(\mathrm{p}-1)}=-\frac{\mathrm{p}^{n}+(n-1) \mathrm{p}^{2}-(3 n-2) \mathrm{p}+2 n-2}{\mathrm{p}^{n}(\mathrm{p}-1)^{2}} .
\end{aligned}
$$

Thus,

(g) $\quad u\left(\frac{1}{n}\right)=-\frac{u(n)}{\mathfrak{X}_{\mathrm{p}}^{2}(n)}=\frac{\left(\mathrm{p}^{n}+(n-1) \mathrm{p}^{2}-(3 n-2) \mathrm{p}+2 n-2\right) \mathrm{p}^{n-2}}{\left(\mathrm{p}^{n-1}+\mathrm{p}-2\right)^{2}}$.

Finally, as $n \rightarrow \infty$, we get $u\left(\frac{1}{n}\right) \rightarrow 1=u(0)$, and thus $u(x)$ is continuous for rational $x$, and we will see that $a$ posteriori it is continuous throughout. This also explains why $\mathcal{S}_{1}(x)$ fails to be continuous: in the above example, $\frac{f_{n}(\mathrm{p})}{g_{n}(\mathrm{p})} \rightarrow_{n \rightarrow \infty} 1$ for $\mathrm{p} \neq 1,|\mathrm{p}-2| \leq 1$; here $f_{n}(\mathrm{p})$ and $g_{n}(\mathrm{p})$ are, respectively, the numerator and the denominator of the r.h.s. of (g). For $\mathrm{p}=1$ we need to apply L'Hôpital's rule to get $\frac{f_{n}^{\prime \prime}(1)}{g_{n}^{\prime \prime}(1)}$, and this, as $n \rightarrow \infty$, tends to $\frac{1}{2}$ rather than to $1=\mathcal{S}_{1}(0)$. 
We use the same notation for $x_{i}$ and $\epsilon_{i}$ as in the previous subsection. Then (国) and (fl) imply

$$
\begin{aligned}
u\left(x_{0}\right) & =-D_{\mathrm{p}}\left(x_{0}\right)-\mathfrak{X}_{\mathrm{p}}^{2}\left(\epsilon_{1}\right) \mathrm{p}^{-a_{0}} u\left(x_{1}\right) ; \text { here } \\
D_{\mathrm{p}}(x) & =\frac{1}{\mathrm{p}} \mathfrak{X}_{\mathrm{p}}(x)+\frac{1}{\mathrm{p}^{2}} \mathfrak{X}_{\mathrm{p}}(x-1)+\cdots+\frac{1}{\mathrm{p}^{n}} \mathfrak{X}_{\mathrm{p}}(x-n+1), \text { if } x \in[n, n+1) ; \\
D_{\mathrm{p}}(x) & =0 \text { if } x \in[0,1) .
\end{aligned}
$$

Thus,

$u(x)=-D_{\mathrm{p}}\left(x_{0}\right)+\mathfrak{X}_{\mathrm{p}}\left(\epsilon_{1}\right)^{2} \mathrm{p}^{-a_{0}} D_{\mathrm{p}}\left(x_{1}\right)-\mathfrak{X}_{\mathrm{p}}\left(\epsilon_{1}\right)^{2} \mathfrak{X}_{\mathrm{p}}\left(\epsilon_{2}\right)^{2} \mathrm{p}^{-a_{0}-a_{1}} D_{\mathrm{p}}\left(x_{2}\right)+\cdots$.

We know that for $|\mathrm{p}-2| \leq 1, \mathrm{p} \neq 1$, the function $\mathfrak{X}_{\mathrm{p}}(x)$ is bounded, and thus so is $D_{\mathrm{p}}(x)$. Therefore, we need to show that the series

(h) $J\left(a_{0}, a_{1}\right)+J\left(a_{0}, a_{1}\right) \cdot J\left(a_{1}, a_{2}\right)+J\left(a_{0}, a_{1}\right) \cdot J\left(a_{1}, a_{2}\right) \cdot J\left(a_{2}, a_{3}\right)+\cdots$,

$$
\text { where } J\left(a_{i}, a_{i+1}\right)=\mathfrak{X}_{\mathrm{p}}\left(\epsilon_{i}\right) \mathfrak{X}_{\mathrm{p}}\left(\epsilon_{i+1}\right)|\mathrm{p}|^{-\left(a_{i}+a_{i+1}\right) / 2},
$$

is absolutely convergent and is majorized by a series which is independent of $x$. If $\epsilon_{i}=[0, a, b, \ldots]$ (here for simplicity we put $a=a_{i}$ and $b=a_{i+1}$ ), then functional equations (ai) imply

$$
\mathfrak{X}_{\mathrm{p}}\left(\epsilon_{i}\right) \mathfrak{X}_{\mathrm{p}}\left(\epsilon_{i+1}\right)=\frac{\mathrm{p}^{a+b}}{\mathrm{p}^{b}+\frac{\mathrm{p}^{a}-1}{\mathrm{p}-1} \mathfrak{X}_{\mathrm{p}}\left(\epsilon_{i+2}\right)+\frac{\mathrm{p}^{a}-1}{\mathrm{p}-1} \cdot \frac{\mathrm{p}^{b}-1}{\mathrm{p}-1}} .
$$

Let $\mathscr{I}_{0}=\mathfrak{X}_{\mathrm{p}}([0,1])$ (see p. 402). We know that $\mathscr{I}_{0}$ is contained within the disc $C_{0}=\left\{z:|z-\mathrm{p}| \leq \frac{4 \mathrm{p}}{3}\right\}$ (p. 401). Since $\epsilon_{i} \in[0,1)$, an absolute convergence of the series (hㅡ) is implied by the following.

Lemma 2. Let $a, b$ be positive integers, and

$$
\sigma_{\mathrm{p}}(a, b)=\sup _{z \in \mathscr{I}_{0}}\left|\frac{\mathrm{p}^{(a+b) / 2}}{\mathrm{p}^{b}+\frac{\mathrm{p}^{a}-1}{\mathrm{p}-1} z+\frac{\mathrm{p}^{a}-1}{\mathrm{p}-1} \cdot \frac{\mathrm{p}^{b}-1}{\mathrm{p}-1}}\right| .
$$

Then

$$
\sigma_{\mathrm{p}}(a, b) \leq 0.7 .
$$

Thus, $|J(a, b)| \leq \sigma_{\mathrm{p}}(a, b)$, and the series (h) is majorized by a geometric series. To prove the lemma, note that if $a$ and $b$ are large, then $\sigma_{\mathrm{p}}(a, b) \sim|\mathrm{p}-1|^{2}$. $|\mathrm{p}|^{-(a+b) / 2}$. Similar estimates hold if one of them is large. Hence, for each $\mathrm{p}$ we need to check only a finite number of cases. Assume $p \in[1,2]$ is real. Then $\mathscr{I}_{0}=[\mathrm{p}-1,1]$, and

$$
\sigma_{\mathrm{p}}(a, b)=\frac{\mathrm{p}^{(a+b) / 2}}{\mathrm{p}^{a}+\mathrm{p}^{b}-1+\frac{\mathrm{p}^{a}-1}{\mathrm{p}-1} \cdot \frac{\mathrm{p}^{b}-1}{\mathrm{p}-1}} \leq \frac{\mathrm{p}^{(a+b) / 2}}{\mathrm{p}^{a}+\mathrm{p}^{b}} \leq \frac{1}{2},
$$

using the arithmetic-geometric mean inequality, and also the fact that $\frac{\mathrm{p}^{a}-1}{\mathrm{p}-1} \geq 1$ for $a \in \mathbb{N}$. The same applies to the case $\mathrm{p} \in[2,3]$ (see [1], Section 2). Thus, the lemma is valid for real $\mathrm{p}$ (and with a sharp constant 0.6 instead of 0.7). By continuity, the lemma is true for a narrow strip around the segment $[1,3]$, intersected with $|p-2| \leq 1$. To verify our lemma for $|p-2| \leq 1$, we use a brute force attack. We know that $\mathscr{I}_{0}$ is contained in $C_{0}$, and thus $\mathscr{I}_{\mathrm{p}}$ is contained in

$$
D_{0}=\bigcup_{n=0}^{\infty}\left(\frac{C_{0}}{\mathrm{p}^{n}}+\frac{\mathrm{p}^{n}-1}{\mathrm{p}^{n}(\mathrm{p}-1)}\right)
$$


Since $\mathscr{I}_{\mathrm{p}}$ is invariant under $z \mapsto \frac{1}{z}, \mathscr{I}_{\mathrm{p}}$ is contained inside $E_{0}=D_{0} \cap D_{0}^{-1}$, and so $\mathscr{I}_{0}$ is contained inside

$$
C_{1}=C_{0} \bigcap \frac{\mathrm{p} E_{0}}{E_{0}+1} .
$$

In the same fashion we get domains $C_{2}, C_{3}$, and so on. This shows that for each fixed $\mathrm{p},|\mathrm{p}-2| \leq 1$, and $\epsilon>0$, there exists $\delta>0$ such that all curves $\mathscr{I}_{\mathrm{p}^{\prime}}$ for $\left|\mathrm{p}-\mathrm{p}^{\prime}\right|<\delta,\left|\mathrm{p}^{\prime}-2\right| \leq 1$, are contained in an $\epsilon$-neighborhood of $\mathscr{I}_{\mathrm{p}}$, and the $\delta-\epsilon$-dependence is explicitly computable. Further, we cover the circle $|\mathrm{p}-2| \leq 1$ with a small grid and verify the validity of the lemma by computer calculations for all grid points. This is a tedious but straightforward job. For a verification of the lemma for a particular $\mathrm{p}$, we use, say, 10 generations of the tree (8). It is easy to get rigorous certificates for all the steps. This proves part (iii). In fact, the maximal value of $\sigma_{\mathrm{p}}(a, b)$ over all $a, b$ and $\mathrm{p},|\mathrm{p}-2| \leq 1$, is apparently $\sigma_{3}(1,1)=0.6$, but to prove this by computation is not that straightforward. In our case computations are easier because we have a reserve. Moreover, for our needs it is enough to show that $\sigma_{\mathrm{p}}(a, b)<0.9$.

1.2. The uniqueness principle for functions in two complex variables claims that if the function vanishes in some 3 -dimensional ball (we consider $\mathbb{C}^{2}=\mathbb{R}^{4}$ ), then the function is identically zero in the whole region of analyticity (contrary to the statement on p. 406, l. 11-12 from above, vanishing on a 2-dimensional ball is obviously not enough: consider $f(\mathrm{p}, z)=\mathrm{p} z)$. In order to show that there exists the unique function which is defined for $|\mathrm{p}-2| \leq 1, z \notin \mathscr{I}_{\mathrm{p}}+1$, and which satisfies the functional equation (22), we must show that a solution of this functional equation is unique for every $p$ on a certain curve inside $|p-2| \leq 1$. As this curve, let us choose the interval $[1,1.5]$. Then our claim follows from:

Proposition 1. Fix real $\mathrm{p}, \mathrm{p} \in[1,1.5]$. Suppose, an analytic function $\mathbf{H}(\mathrm{p}, z)$ is defined in the domain $\mathbb{C} \backslash\left(\mathscr{I}_{\mathrm{p}}+1\right), \mathbf{H}(\mathrm{p}, z) \rightarrow 0$ if $\operatorname{dist}\left(z, \mathscr{I}_{\mathrm{p}}\right) \rightarrow \infty$, and it satisfies the functional equation

$$
\frac{\mathrm{p}}{z^{2}} \mathbf{H}\left(\mathrm{p}, \frac{\mathrm{p}}{z}\right)+2 \mathbf{H}(\mathrm{p}, z+1)=\mathrm{pH}(\mathrm{p}, \mathrm{p} z), \text { for } z \notin \mathscr{I}_{\mathrm{p}} .
$$

Then $\mathbf{H}(\mathrm{p}, z) \equiv 0$.

Proof. As in the proof of Lemma 6.2, let us consider $\widehat{\mathbf{H}}(z)=\mathbf{H}(\mathrm{p},-z)$. In the above equation, substitute $z \mapsto \mathrm{p}^{n} z-\frac{\mathrm{p}^{n+1}-1}{\mathrm{p}-1}$, multiply by $\mathrm{p}^{n} 2^{-(n+1)}$ and sum over $n \geq 0$. This gives the series

$$
\widehat{\mathbf{H}}(z)=-\sum_{n=0}^{\infty} \frac{\mathrm{p}^{n+1}}{2^{n+1}\left(\mathrm{p}^{n} z+\frac{\mathrm{p}^{n+1}-1}{\mathrm{p}-1}\right)^{2}} \widehat{\mathbf{H}}\left(\frac{\mathrm{p}}{\mathrm{p}^{n} z+\frac{\mathrm{p}^{n+1}-1}{\mathrm{p}-1}}\right) .
$$

Consider this for $z \in[0, \mathrm{p}]$. All arguments on the right belong to this interval as well. Let $z_{0} \in[0, \mathbf{p}]$ be such that $M=\left|\widehat{\mathbf{H}}\left(z_{0}\right)\right|=\sup _{z \in[0, \mathrm{p}]}|\widehat{\mathbf{H}}(z)|$. Consider the above expression for $z=z_{0}$. Thus,

$$
M=\left|\widehat{\mathbf{H}}\left(z_{0}\right)\right| \leq M \sum_{n=1}^{\infty} \frac{\mathrm{p}^{n}(\mathrm{p}-1)^{2}}{2^{n}\left(\mathrm{p}^{n}-1\right)^{2}} \leq 0.9 \cdot M \text { for } \mathrm{p} \in[1,1.5] .
$$

As before, this is contradictory unless $M=0$. 
1.3. Let $\mathbb{Q}[z]_{n-1}$ denote the linear space of dimension $n$ of polynomials of degree $\leq$ $n-1$ with rational coefficients. Consider the following linear map $\mathcal{L}_{n-1}: \mathbb{Q}[z]_{n-1} \mapsto$ $\mathbb{Q}[z]_{n-1}$, defined by (p. 408)

$$
\mathcal{L}_{n-1}(P)(z)=P(z+1)-\frac{1}{2^{n+1}} P(2 z)+\frac{(-1)^{n+1}}{2^{n+1}} P\left(\frac{2}{z}\right) z^{n-1} .
$$

Lemma 6.1 claims that $\operatorname{det} \mathcal{L}_{n-1} \neq 0$. To better understand the structure of denominators of rational numbers contained in the tables on pp. 388-389, we need the following refinement. (This was stated without a proof on p. 408 and referred to on p. 389 , l. 15 from below, also).

Proposition 2. Let $m=\left\lfloor\frac{n}{2}\right\rfloor$. Then $\operatorname{det}\left(\mathcal{L}_{n-1}\right)=\frac{\prod_{i=1}^{m}\left(4^{i}-1\right)}{2^{m^{2}+m}}$.

Proof. First, the value of this determinant does not depend on a basis of the linear space $\mathbb{Q}[z]_{n-1}$. As the basis, let us choose $\left\{1, z, z^{2}, \ldots, z^{n-1}\right\}$. Consider also the linear map $\mathcal{K}_{n-1}: \mathbb{Q}[z]_{n-1} \mapsto \mathbb{Q}[z]_{n-1}$, defined by $\mathcal{K}_{n-1}(P)(z)=P(z-1)$. Consider the map $\mathcal{T}=\mathcal{K}_{n-1}^{-1} \circ \mathcal{L}_{n-1} \circ \mathcal{K}_{n-1}^{2}$. Then by direct calculation

$$
\mathcal{T}(P)(z)=P(z)-\frac{1}{2^{n+1}} P(2 z)+\frac{(-1)^{n+1}}{2^{n+1}} P\left(-\frac{2 z}{z+1}\right)(z+1)^{n-1} .
$$

In particular,

$$
\mathcal{T}\left(z^{i}\right)=z^{i}-2^{i-n-1} z^{i}+(-1)^{n+i+1} 2^{i-n-1} z^{i}(z+1)^{n-i-1}, \quad 0 \leq i \leq n-1 .
$$

Thus, the matrix representation of $\mathcal{T}$ in the basis $\left\{1, z, \ldots, z^{n-1}\right\}$ is lower triangular with diagonal entries $1-2^{i-n-1}+(-1)^{n+i+1} 2^{i-n-1}$, and so

$$
\begin{aligned}
\operatorname{det}\left(\mathcal{K}_{n-1}\right)^{-1} \cdot \operatorname{det}\left(\mathcal{L}_{n-1}\right) \cdot \operatorname{det}\left(\mathcal{K}_{n-1}\right)^{2} & =\operatorname{det}\left(\mathcal{K}_{n-1}^{-1} \circ \mathcal{L}_{n-1} \circ \mathcal{K}_{n-1}^{2}\right) \\
& =\prod_{i=0}^{n-1}\left(1-2^{i-n-1}+(-1)^{n+i+1} 2^{i-n-1}\right) .
\end{aligned}
$$

Finally, it is obvious that $\operatorname{det}\left(\mathcal{K}_{n-1}\right)=1$. A simple calculation shows that the number on the right is exactly the magnitude in the formulation of the proposition.

Naturally, we cannot claim that eigenvalues of $\mathcal{L}_{n-1}$ itself are that simple. For example, if $n=6$, the characteristic polynomial of $\mathcal{L}_{5}$ is

$$
\lambda^{6}-\frac{705}{128} \lambda^{5}+\frac{104667}{8192} \lambda^{4}-\frac{4025189}{262144} \lambda^{3}+\frac{2927697}{262144} \lambda^{2}-\frac{491967}{131072} \lambda+\frac{2835}{4096},
$$

which is irreducible over $\mathbb{Q}$.

1.4. Our main Theorem 1.2 claims that the dyadic period function is an infinite sum of rational functions with rational coefficients: for $\left\{|z| \leq \frac{3}{4}\right\} \cup\left\{\left|z+\frac{9}{7}\right| \leq \frac{12}{7}\right\}$ there exists polynomials $\mathscr{B}_{n}(z) \in \mathbb{Q}[z]_{n-1}$, such that

$$
G(z)=\sum_{n=0}^{\infty}(-1)^{n} \frac{\mathscr{B}_{n}(z)}{(z-2)^{n+1}} .
$$

As a matter of fact, employing directly the Calkin-Wilf tree (2) and the functional equation (12), 2], one can derive another such series of a completely different nature. On the one hand, the Corollary 1.4 and Experimental Observation 1.6 give some mysterious series for the moments of the Minkowski question mark function. 
In the case to follow, the series for the moments so obtained is a tautological statement equivalent to the formula on p. 385, l. 6 from above.

If $\frac{a}{b}$ is a positive rational number and $\frac{a}{b}$ belongs to the $n$th generation of the Calkin-Wilf tree (2) (thus, $\frac{1}{1}$ belongs to the first generation), let $\chi\left(\frac{a}{b}\right)=2^{-n}$.

Proposition 3. For $z \in \mathbb{C} \backslash(0, \infty)$, we have an absolutely convergent series

(i) $\quad G(z+1)=\int_{0}^{\infty} \frac{1}{x-z} \mathrm{~d} F(x)=\sum_{\substack{a, b, c, d \geq 0, a d-b c=1}} \frac{\chi\left(\frac{a+b}{c+d}\right)}{[(a+c) z-(b+d)](c z-d)}$.

Proof. The functional equation (12), 2] can be rewritten as

$$
2 G(z+1)=G(z-1+1)+\frac{1}{(1-z)^{2}} G\left(\frac{z}{1-z}+1\right)+\frac{1}{1-z}
$$

Consider two Möbius transformations:

$$
T^{-1}(z)=z-1, \quad R^{-1}(z)=\frac{z}{1-z} .
$$

Let the semigroup $\Gamma$ be generated by $T^{-1}$ and $R^{-1}$. Then

$$
\Gamma=\left\{\left(\begin{array}{cc}
a & -b \\
-c & d
\end{array}\right): a d-b c=1, a, b, c, d \in \mathbb{N}_{0}\right\} .
$$

Inside the group $\mathrm{SL}_{2}(\mathbb{Z})$, all relations between $T$ and $R$ are given by $\left(T \circ R^{-1}\right)^{3}=I$ and $\left(T \circ R^{-1} \circ T\right)^{2}=I, I$ being the identity matrix. Hence $\Gamma$ is a free semigroup. If $\gamma \in \Gamma$ and $\gamma$ is a product of $n$ copies of $R^{-1}$ or $T^{-1}$, let $S(\gamma)=2^{-n}$. Further, consider the standard weight 2 "slash" operator, given by

$$
\left.f(z)\right|_{\gamma}=\frac{1}{(c z+d)^{2}} f\left(\frac{a z+b}{c z+d}\right), \quad \gamma=\left(\begin{array}{ll}
a & b \\
c & d
\end{array}\right), \quad \gamma \in \mathrm{SL}_{2}(\mathbb{Z})
$$

Finally, note that

$$
\Gamma=\Gamma \cdot T^{-1} \cup \Gamma \cdot R^{-1} \cup\{I\} .
$$

If we compare (j] $)$ with the decomposition $(\mathrm{k})$ and use the uniqueness property of $G(z)([2$, Section 2) we immediately obtain the series

$$
G(z+1)=\left.\frac{1}{2} \sum_{\gamma \in \Gamma} S(\gamma) \cdot \frac{1}{1-z}\right|_{\gamma}
$$

This is exactly the series (ii). It is absolutely convergent. Indeed, let $z \in \mathbb{C} \backslash(0, \infty)$. For every pair of integers $(c, d)=1, c \geq 0, d \geq 1$, let $\left(c^{\prime}, d^{\prime}\right)$ be the smallest nonnegative pair such that $c^{\prime} d-c d^{\prime}=1$. Note that $\chi\left(\frac{c^{\prime}+d^{\prime}}{c+d}+n\right)=2^{-n} \chi\left(\frac{c^{\prime}+d^{\prime}}{c+d}\right)$. Thus, the series (ii) is majorized by

$$
\begin{array}{r}
\sum_{(c, d)=1} \sum_{n=1}^{\infty} \frac{2^{-n+1} \chi\left(\frac{c^{\prime}+d^{\prime}}{c+d}\right)}{\mid\left(c^{\prime} z-d^{\prime}+n(c z-d)|\cdot| c z-d \mid\right.} \\
\quad=\sum_{(c, d)=1} \sum_{n=1}^{\infty} \frac{2^{-n+1} \chi\left(\frac{c^{\prime}+d^{\prime}}{c+d}\right)}{|\gamma(z)-n| \cdot|c z-d|^{2}} .
\end{array}
$$

Here $\gamma(z)=\frac{c^{\prime} z-d^{\prime}}{-c z+d}$. Since the cusp of a Möbius transformation $\gamma$ for $c \neq 0$ is $-\frac{c^{\prime}}{c}$ and it is contained in the interval $[-1,0]$, the standard tessellation of the 
upper half-plane by the modular group shows that $\Re(\gamma(z))$ is uniformly bounded independently of $c, d$ and any $z$ in any compact set inside $\mathbb{C} \backslash[0, \infty)$. The series $\sum_{n} \frac{2^{-n+1}}{n}$ converges, so we need to show that

$$
\sum_{(c, d)=1} \frac{\chi\left(\frac{c^{\prime}+d^{\prime}}{c+d}\right)}{|c z-d|^{2}},
$$

absolutely converges, which is obvious: for $n \geq 2$, there are $2^{n-2}$ rational numbers $\frac{c^{\prime}+d^{\prime}}{c+d}<1$ in the $n$th generation of the Calkin-Wilf tree, and if $\frac{c^{\prime}+d^{\prime}}{c+d}$ belongs to the $n$th generation, then $c+d \geq n$. Further, we write $z=x+i y$ and compare the above series with $M \cdot \sum_{n} n^{-2}$ for a certain $M=M(x, y)$.

\section{Corrigenda}

To disambiguate, we note that throughout the paper, $\frac{\mathrm{p} \mathscr{I}_{\mathrm{p}}}{\mathscr{I}_{\mathrm{p}}+1}$ stands for

$$
\bigcup\left\{\frac{\mathrm{p} x}{x+1}: x \in \mathscr{I}_{\mathrm{p}}\right\} \text {. }
$$

p. 394, 1. 8-9 from below. The formulas should read as

$$
\begin{aligned}
& \frac{1}{\mathrm{p}-1} \leq x \leq \mathrm{p}-1 \quad \Leftrightarrow \quad 1 \leq \frac{\mathrm{p} x}{x+1} \leq \mathrm{p}-1, \\
& \frac{1}{\mathrm{p}-1} \leq x \leq \mathrm{p}-1 \quad \Leftrightarrow \quad \frac{1}{\mathrm{p}-1} \leq \frac{x+1}{\mathrm{p}} \leq 1 .
\end{aligned}
$$

p. 399, 1. 11 from below. Read "(a) or (b)" instead of "(a) and (b)".

p. 399, 1. 6 from below. Read $W_{a}(\mathrm{p}) \rightarrow \frac{1}{\mathrm{p}-1}$.

p. 401, 1. 4 from above. Read $\frac{A_{N+2}^{\prime}}{B_{N+2}^{\prime}}$.

p. 403, l. 4 from above. Replace $B_{0, T}$ with $B_{0, T}(\mathrm{p})$.

p. 403, 1. 5 from below. Read $\left|M_{L}(\mathrm{p})\right|$ instead of $M_{L}(\mathrm{p})$.

p. 405, 1. 9 from below. Replace $z \notin \frac{\mathscr{I}_{\mathrm{p}}+1}{\mathrm{p}}$ with $z \notin \mathscr{I}_{\mathrm{p}}$. Note that, as remarked on p. $396, \frac{\mathscr{I}_{\mathrm{p}}+1}{\mathrm{p}} \subset \mathscr{I}_{\mathrm{p}}$.

p. 415,1 . 5 from above. Read $\mathrm{C}=e^{-2 \sqrt{\log 2}}$ instead of $\mathrm{C}=e^{-2} \sqrt{\log 2}$.

Though the formulas in the Section 6 (starting from p. 407) and Appendix A.1 are correct, there is a confusion in not distinguishing the notation for partial derivative and full derivative. For example, the right-hand side of (24) is using a correct notation, while the left-hand side should read as

$$
\frac{\mathrm{d}^{n}}{\mathrm{~d}^{n}}(\mathrm{p} G(\mathrm{p}, \mathrm{p} z))
$$

We hope that this imprecision in notation does not confuse the reader.

We note that, though the Experimental Observation 1.5 on p. 388 might truly hold, but, unlike it is stated in the main paper, we do not have enough numerical data 
to claim its validity. Since the denominators of rational numbers $\mathbf{H}_{n}^{(L-1)}(1)$ grow very fast, the standard home computer can handle them up to $n=60$. As stated before, the Experimental Observation 1.5 does hold for $L=1$, and it is likely to be true for $L=2$. For $L \geq 3$, we do not have decisive evidence yet.

\section{ACKNOWLEDGEMENT}

The author thanks the referee and also Gerald Kuba whose remarks helped to shorten some proofs. The author gratefully acknowledges support from the Austrian Science Fund (FWF) under the project Nr. P20847-N18.

\section{REFERENCES}

[1] G. Alkauskas, The Minkowski question mark function: explicit series for the dyadic period function and moments, Math. Comp. 79 (269) (2010), 383-418. MR2552232 (2010k:11006)

[2] G. Alkauskas, The moments of Minkowski question mark function: the dyadic period function, Glasg. Math. J. 52 (1) (2010), 41-64. MR2587817

[3] H. S. WaLl, Analytic theory of continued fractions, D. Van Nostrand Company, Inc., New York, N. Y., 1948. MR0025596(10:32d)

Institute of Mathematics, Department of Integrative Biology, Universität für Bodenkultur Wien, Gregor Mendel-Strasse 33, A-1180 Wien, Austria, and Vilnius University, Department of Mathematics and Informatics, Naugarduko 24, LT-03225 Vilnius, LITHUANIA

E-mail address: giedrius.alkauskas@gmail.com 